\title{
Eliminating Target Cells by Inducing Apoptosis-Related Factors Using hTERT Promoter
}

\author{
Tomoki Takashina $^{\mathrm{a}}$ and Manabu Nakayama*,a,b
}

\author{
${ }^{a}$ Laboratory of Pharmacogenomics, Graduate School of Pharmaceutical Sciences, Chiba University, 2-6-7 Kazusa- \\ Kamatari, Kisarazu, Chiba 292-0818, Japan \\ ${ }^{b}$ Department of Human Genome Research, Kazusa DNA Research Institute, 2-6-7 Kazusa-Kamatari, Kisarazu, Chiba \\ 292-0818, Japan
}

\begin{abstract}
Inducing the complete apoptosis cascade in target cells is useful for eliminating cancer cells and for producing animal models lacking specific cell types. Neuroblastoma SH-SY5Y cells lack caspase-8 and are thus resistant to the apoptosis-inducing effects of 2DEDplusE, an engineered Fas-associated death domain protein (FADD) containing tandem death effector domains (DEDs) of FADD and lambda phage E protein. To overcome tumor cells' resistance to apoptosisinducing factors, we produced new engineered factors-2DEDcasp8, 2DEDcasp8 $\triangle D E D a$, and 2DEDcasp8 $\Delta$ DEDab—by fusing the tandem DEDs of FADD to procaspase- 8 or its different segments. Of the three, 2DEDcasp8 $\triangle D E D a$ most effectively induced apoptosis in SH-SY5Y cells. Finally, by using the human telomerase reverse transcriptase (hTERT) promoter, a known cancer cell-specific promoter, and adapting it to a mifepristone-inducing system, we expressed engineered factors that induced apoptosis in HeLa and A549 tumor cells. The same system did not induce apoptosis in normal Wi-38 and MRC-5 cells, although driving the same system with a constitutive thymidine kinase (TK) promoter induced apoptosis in both cell types.
\end{abstract}

Keywords: Apoptosis, FADD, Biotechnology, Engineered protein, Human telomerase reverse transcriptase.

\section{INTRODUCTION}

The engineered Fas-associated death domain protein (FADD), 2DEDplusE, which was made by fusing the tandem DEDs of FADD to the E protein of lambda phage, greatly enhances apoptosis-inducing activity in adherent cells in vitro [1]. The engineered factor produced artificial deathinducing signaling complex (DISC)-like signals in the cytoplasm that appear as grains under fluorescence microscopy [2]. Membrane blebbing associated with apoptosis was observed just after formation of grain-like signals. Immunoprecipitation analysis demonstrated that 2DEDplusE-FLAG can bind $\mathrm{p} 43 / \mathrm{p} 41$ forms of caspase-8, but E protein-FLAG cannot. Gel filtration analysis demonstrated that 2DEDplusE forms a large complex containing partially cleaved procaspase-8 (p43/p41) in the cytoplasm; the size of this complex varies greatly. The engineered FADD complex closely mimics intrinsic DISC and increases apoptosis-inducing activity.

Apoptotic defects resulting from epigenetic alterations and loss of heterozygosity are believed to be responsible for tumor formation, progression, and resistance to anti-cancer drugs [3, 4]. Various types of cancer cells use different strategies to escape the apoptosis cascade. Our aim is to develop a reliable means of inducing the complete apoptosis cascade in a given targeted cell type. In this study, we examined whether our engineered factors can induce apoptosis in

\footnotetext{
*Address correspondence to this author at the 2-6-7 Kazusa-Kamatari, Kisarazu, Chiba 292-0818, Japan; Tel: +81-438-52-3909; Fax: +81-438-523931; E-mail: nmanabu@kazusa.or.jp
}

various types of cancer cells and in potentially more resistant cells originating from human tumors. We also examined whether a tumor-specific promoter can be used to express the engineered factors and specifically induce apoptosis in tumor cells.

\section{MATERIALS AND METHODS}

\section{Construction of Expression Vector for Producing Engi- neered Protein}

The construction of 2DEDplusE was described previously [1]. Briefly, tandem DEDs of FADD were fused to the E protein of lambda bacteriophage. We constructed 2DEDcasp8 by fusing the tandem DEDs (amino acid (aa) positions 1 to 95) of mouse FADD to human procaspase-8. Next, we constructed 2DEDcasp $8 \triangle D E D a$ by fusing $2 D E D$ to a part of procaspase- 8 (aa positions 85 to 480 , the end portion of procaspase-8), and then removed the first DED (also called DEDa). Finally, we constructed 2DEDcasp8 $\triangle$ DEDab by fusing 2DED to a part of procaspase- 8 (aa positions 181 to 480 ), and then removed both DEDs (i.e., DEDa and $\mathrm{DEDb}$ ).

We constructed plasmid pSwitch-neo by replacing the ampicillin resistance gene of pSwitch (Invitrogen, CA, USA) with the neomycin resistance gene of pMC1neoPolyA (Stratagene, CA, USA). Oligomers (5'-CGCACGTGATGG GATCGGCCATTGAACAA-3', 5'-CGCACGTGTCAGA AGAACTCGTCAAGAAG-3') were used for the PCR amplification of the neomycin resistance gene. The resulting PCR product was digested with PmII. We ligated the DNA fragment onto the PmlI fragment of pSwitch. For construction of pGeneSwitch, the pGene/V5-HisB plasmid was di- 
gested with AatII and PvuII and blunted, and the 900-bp AatII-PvuII DNA fragment was ligated into the blunted Sse8387I site of pSwitch-neo. For construction of pGeneSwitch-EGFP, the pGeneSwitch plasmid was digested with ApaLI and NsiI and blunted, and the ApaLI-NsiI DNA fragment was ligated into the blunted AseI site of pEGFP-C3 (Clontech).

\section{Construction of pGeneSwitch-EGFP-hTERT Vector for Tumor-Specific Expression}

The human telomerase reverse transcriptase (hTERT) promoter region ( -475 to +49 ) was amplified by PCR using the following primers: sense, 5'-GGGATCGATCTCCC CTTCACGTCCGGCATT-3'; and antisense, 5'-CCCTTAA TTAACGGGGCCAGGGCTTCCCACGT-3', as described previously [5]. The resulting PCR product was digested with BspDI and PacI and blunted. We ligated the DNA fragment onto the BspDI and PacI fragment of pSwitch (Invitrogen) in place of the thymidine kinase (TK) promoter, and named the plasmid pSwitch hTERT. The pSwitch hTERT plasmid was digested with NruI and AsnI, and the NruI-AsnI DNA fragment was ligated into the NruI-AsnI site of pGeneSwitch EGFP. The resultant plasmid was named pGeneSwitchEGFP-hTERT.

\section{Cell Culture Conditions}

SH-SY5Y neuroblastoma cells were obtained from American Type Cell Collection (ATCC, Manassas, VA, USA), and were grown in a mixture (1:1) of Basal Medium Eagle (BME; Invitrogen) and Ham's F-12 (F-12; Invitrogen) containing $10 \%$ fetal bovine serum (FBS). Wi-38 and MRC5 cells, both normal human fetal lung fibroblast cell lines, were obtained from Japanese Collection of Research Bioresources (JCRB) and were grown with BME containing 10\% FBS. Flp-in HEK293 cells were obtained from Invitrogen.

\section{Quantitative Real-Time PCR (qPCR) Analysis of hTERT mRNA in Various Cells}

Total RNA was purified from Wi-38, MRC-5, HeLa, and A549 cells using RNeasy Mini Kit (QIAGEN). A549 is a human lung adenocarcinoma epithelial cell line. For each cell line, $100 \mathrm{ng}$ of total RNA were reverse transcribed using SuperScript III Platinum SYBR Green One-Step qRT-PCR Kit (Invitrogen) and gene-specific primers. The following gene-specific primers were used: sense, 5'-CACGCGA AAACCTTCCTCA-3 and antisense, 5'-CAAGTTCACCAC GCAGCC-3' for human TERT; and sense, 5'-TCCTTCCTG GGCATGGAG-3' and antisense, 5'-AGGAGGGAGCAA TGATCTTGATCTT-3' for $\beta$-actin. PCR reactions were carried out at $50^{\circ} \mathrm{C}$ for $5 \mathrm{~min}, 95^{\circ} \mathrm{C}$ for $2 \mathrm{~min}$ for the reversetranscription step, 40 cycles at $95^{\circ} \mathrm{C}$ for $3 \mathrm{sec}$ and $60^{\circ} \mathrm{C}$ for $30 \mathrm{sec}$ for amplification, and then $95^{\circ} \mathrm{C}$ for $15 \mathrm{sec}, 60^{\circ} \mathrm{C}$ for $1 \mathrm{~min}, 95^{\circ} \mathrm{C}$ for $15 \mathrm{sec}$, and $60^{\circ} \mathrm{C}$ for $15 \mathrm{sec}$. Quantitative PCR was performed with a 7500Fast Real-Time PCR System (Applied Biosystems, Foster City, CA, USA).

\section{Quantitative Analysis of Cell Death}

The genes of a series of engineered proteins were inserted into the SpeI site of pGeneSwitch-EGFP vector. DNA transfection into cells was performed using Nupherin (BIOMOL international, Inc., Plymouth Meeting, PA, USA) or FuGENE HD Transfection Reagent (Roche). Twenty-four hours after transfection, the photos were recorded using fluorescence microscopy (IX71, Olympus) and ORCA-ER, and then the number of cells with EGFP signals was counted. Two, 6, 12, and 24 hours after mifepristone treatment, photos of the cells were taken, and then the number of healthy cells with EGFP signals was counted. Cells undergoing apoptosis, namely blebbing cells or fragmented cells, were not counted. Cell survival was represented as a ratio of the number of surviving cells at each time point after mifepristone induction to the number of cells expressing EGFP before induction. Typically, we counted over 300 cells per experiment.

Caspase-8, 2DEDcasp8, 2DEDcasp8 $\triangle$ DEDa, 2DEDcasp $8 \triangle \mathrm{DEDab}$, and 2DEDplusE genes were inserted into pGeneSwitch-EGFP-hTERT and pGeneSwitch-EGFP vectors, the latter of which was driven by the TK promoter. The vectors were then transfected into Wi-38, MRC-5, A549, and HeLa cells.

\section{RESULTS}

\section{Neuroblastoma SH-SY5Y Cells Lacking Caspase-8 are Resistant to the Apoptosis-Inducing Effects of 2DEDplusE}

We previously demonstrated that 2DEDplusE, an engineered FADD, bound innate procaspase-8, formed DISC complexes, and then activated caspase- 8 with self-activating activity $[1,2]$. The activated caspase- 8 also induced the activation of procaspase-3. Clearly, 2DEDplusE induces caspase-dependent apoptosis mainly through the caspase-8to-caspase- 3 cascade. One of the long-term goals of our laboratory is to develop a method that specifically and selectively eliminates human cancer cells. Because various types of cancer cells are known to escape the apoptosis cascade through different strategies [3, 4], we wondered whether cells lacking caspase- 8 are resistant to 2DEDplusE-induced apoptosis. However, because certain cancer cells possess caspase-10, a paralogue of caspase-8, 2DEDplus may still induce apoptosis in these cells [6]. To distinguish these two possibilities, we tested the effects of 2DEDplusE on neuroblastoma SH-SY5Y cells, which do not express caspase-8 due to the epigenetic gene silencing of the caspase- 8 gene locus through methylation [7].

Because the transfection efficiency of SH-SY5Y cells is relatively lower than that of HEK293 and NIH3T3 cells, we counted the transfected cells first and then counted the surviving cells after 2DEDplusE induction with mifepristone. Most of the transfected cells expressing the EGFP marker protein survived 2 and 6 hours after 2DEDplusE induction (Fig. 1). These results strongly suggested that 2DEDplusE induced apoptosis via caspase-8, because the SH-SY5Y cells, which lack caspase-8, resisted 2DEDplusE-dependent apoptosis. This is consistent with our proposed mechanism that 2DEDplusE induces apoptosis in cells. This result also revealed one critical problem: Tumor cells lacking caspase- 8 are resistant to 2DEDplusE-induced apoptosis. Thus, we tried to develop other types of engineered proteins capable of inducing apoptosis even in SH-SY5Y cells.

\section{New Engineered Factors can Overcome Tumor Cells' Resistance to Apoptosis-Inducing Factors}

As we constructed another series of engineered proteins (Fig. 2A), we took the following three issues into account: 

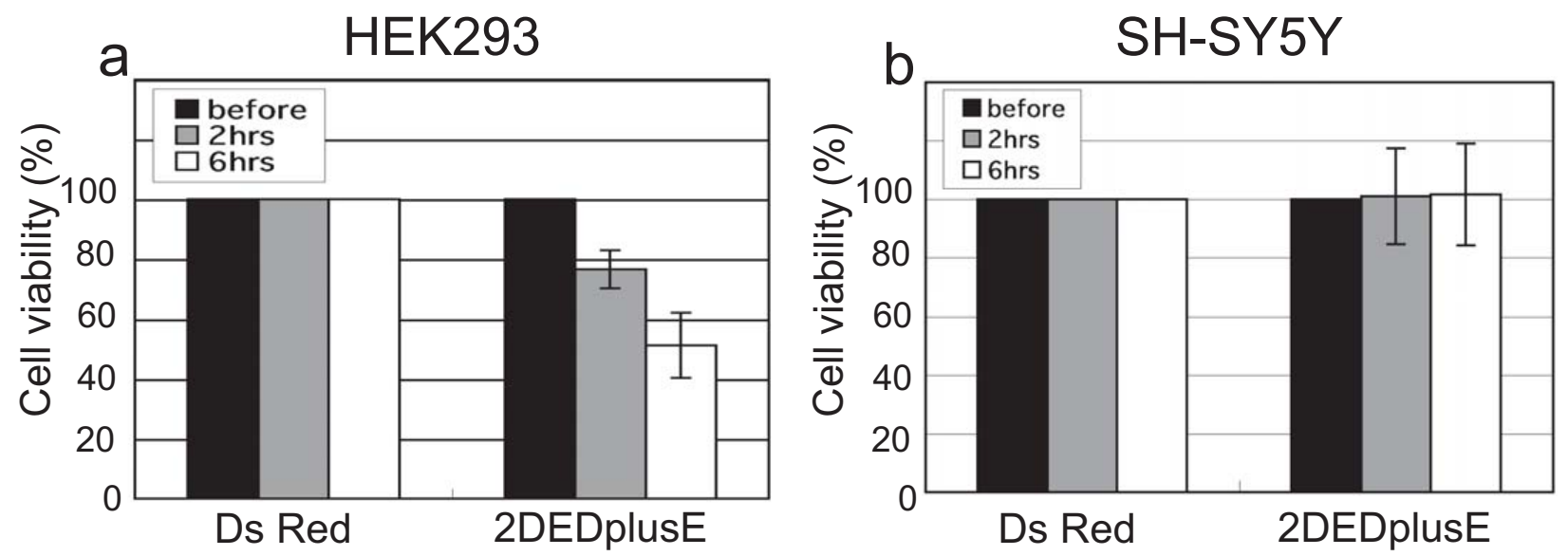

Fig. (1). Effects of 2DEDplusE against cells that express or do not express caspase-8. The bar graphs show the ratios of surviving cells 2 and 6 hours after 2DEDplusE induction. Data were normalized according to the number DsRed-expressing cells (negative control). The DsRedexpressing cells did not express any of the engineered factors.

(1) lack of caspase-8 in cancer cells; (2) apoptosis-inducing activity of DED domains; and (3) final size of the engineered protein. Firstly, we ensured that the new engineered proteins contained caspase- 8 in the form of procaspase- 8 , which was composed of two DED domains (DEDa and DEDb) and p18 and $\mathrm{p} 10$. After activation of self-proteolytic activity, these DEDs were removed to generate a heterodimeric protease enzyme consisting of p18 and p10. Essentially, p18 and p10 are necessary for the activity of activated caspase-8. Secondly, we used tandem domains of FADD rather than 2DEDplusE. Our previous results showed that the tandem DEDs of 2DEDplusE contributed most to the enhancement of apoptosis, whereas the E protein contributed only moderately [2]. Thirdly, we endeavored to make the size of the engineered proteins as small as possible. Our previous experience indicated that most large proteins cannot be produced in cells abundantly for the following three reasons: (1) Large proteins tend to be unstable; (2) large proteins possess many sites where internal proteinases can attack; and (3) large protein-associated translational machinery is less efficient. With these three issues in mind, we constructed another series of engineered proteins (Fig. 2A): 2DEDcasp8, 2DEDcasp8 $\triangle \mathrm{DEDa}$, and 2DEDcasp8 $\triangle \mathrm{DEDab}$.

The 2DEDcasp8 protein was composed of tandem DEDs from FADD and full-length procaspase-8. The 2DEDcasp8 $\triangle$ DEDa protein was constructed by removing DEDa of procaspase-8 from 2DEDcasp8. Because DEDb of procaspase- 8 was bound to DED of FADD, we kept DEDb but removed DEDa in order to reduce the size of the final protein. The 2 DEDcasp $8 \triangle D E D a b$ protein was constructed by removing both DEDa and DEDb of procaspase- 8 . We expected the tandem DEDs of FADD would replace the function carried out by DEDa and DEDb of procaspase- 8 . FADD is composed of a single DED and a single DD. We did not construct a protein composed of 2DEDplsuE and procaspase- 8 , because it was too large in size.

Next, we tested the apoptosis-inducing activity of these three new proteins in SH-SY5Y cells. Recall that almost no apoptosis activity was observed when 2DEDplusE was expressed in SH-SY5Y cells and induced with mifepristone. By contrast, apoptosis activity was induced to $40 \%$ and $50 \%$ in SH-SY5Y cells transfected with either 2DEDcasp8 or 2DEDcasp8 $\triangle D E D a$, respectively. However, apoptosis activity was induced to only $20 \%$ and $10 \%$ in SH-SY5Y cells transfected with either procaspase-8 (casp8) or 2DEDcasp8 $\triangle$ DEDab, respectively (Fig. 2B). These results showed that 2DED of FADD enhanced apoptosis activity of procaspase-8. It should be noted, however, that the TK promoter of the GeneSwitch system used in the present study is weaker than the TET promoter of the T-REx system, which was used in our previous study [1]. Thus, if a stronger promoter is used to express these novel engineered factors, then we should expect to see a higher rate of apoptosis in transfected cells. In summary, 2DEDcasp $8 \triangle \mathrm{DEDa}$ was the strongest apoptosis-inducing factor among the three novel engineered proteins. It induced apoptosis in cells that lacked either caspase- 8 or caspase-3.

\section{Tumor-Specific Expression of Engineered Factors with the hTERT Tumor-Specific Promoter}

Since the newly engineered factors induced apoptosis in some human tumor cells, especially apoptosis-resistant cells lacking caspase- 8 , this prompted us to determine whether the engineered factors can be expressed in a tumor-specific manner. We specifically selected the hTERT promoter to drive the expression of the factors. Telomerase maintains telomere ends by adding telomere repeats and consists of a reverse transcriptase and an RNA component. Telomerase expression also plays a role in cellular senescence. Postnatal somatic cells repress TERT expression, which results in progressive shortening of telomeres. Most tumor cells are believed to express high levels of TERT, resulting in endless cell replication [8]. Thus, hTERT promoter is often used to drive the expression of genes for tumor-specific expression. We constructed a tumor-specific expression vector carrying the hTERT promoter, which directed GAL4DBD/hPRLBD/p65-AD (Fig. 3A). This expression system is based on the GeneSwitch system. GAL4DBD/hPR-LBD/p65-AD binds GAL4UAS, which is located adjacent to the mifepristone-dependent gene of interest. In the presence of mifepristone, the gene of interest is then transcriptionally expressed. GAL4DBD/hPR-LBD/p65-AD also binds to the distal region of hTERT and can amplify GAL4DBD/hPR-LBD/p65-AD 

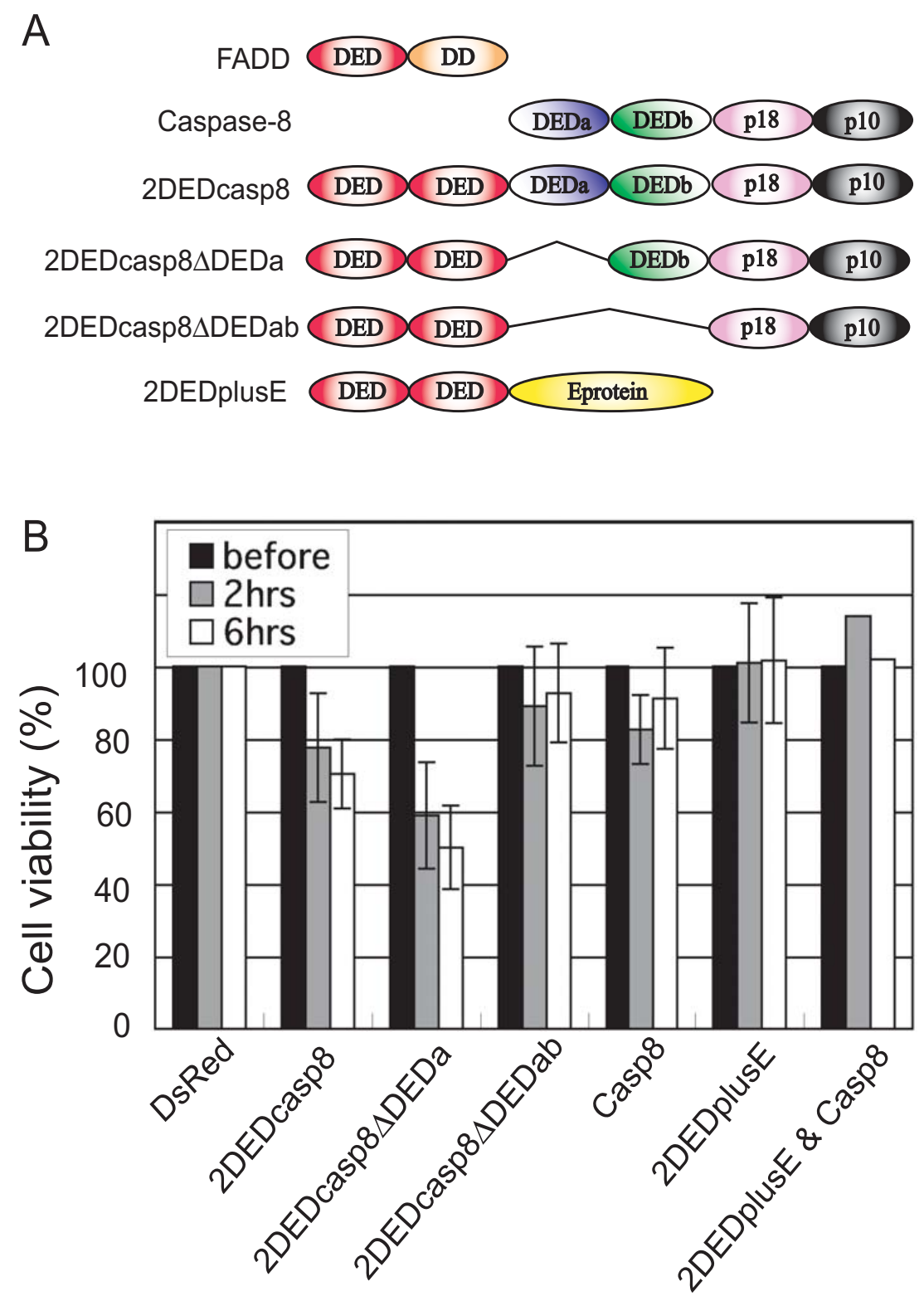

Fig. (2). (A) Domain organization of three novel engineered factors. These factors were based on a factor containing tandem DEDs of FADD and full-length procaspase-8. DEDa and DEDb represent the first DED and second DED of procaspase-8, respectively; p18 and p10 represent the large and small subunits of caspase-8, respectively. E protein is a lambda bacteriophage head protein. (B) Cell viability of SH-SY5Y cells expressing different novel engineered proteins. Bar graph showing the ratios of surviving cells 2 and 6 hours after the induction of the engineered proteins, reflecting the cell death activity of the engineered proteins. Of the proteins analyzed, 2DEDcasp8 $\Delta D E D a$ showed the strongest cell death activity in SH-SY5Y neuroblastoma cells, cells that do not express caspase-8.

itself. This expression vector also contains an EGFP gene, which is directed by a constitutive CMV promoter. EGFP serves as a marker for transfected cells. For constitutive expression after adding mifepristone, the TK promoter is used in place of hTERT.

To confirm previous findings that hTERT expression is low in normal cells but high in cells originating from tumors, we compared hTERT expression in normal cells (Wi-38 and MRC-5 cells) with that in tumor cells (HeLa and A549 cells) using qPCR. Our qPCR results were consistent with those reported previously: Normal cells expressed low levels of
hTERT, whereas cells originating from tumors expressed high levels of hTERT (Fig. 3B). Thus, we expected that the hTERT promoter could drive the expression of a gene of interest in a tumor-specific manner, as described previously [5]. Tumor-specific expression was confirmed by expression of EGFP in the transfected cells (data not shown). When the expression vector containing the hTERT promoter was introduced into tumor cells, brilliant DsRed signals were detected in over 60 to $70 \%$ of the EGFP-positive cells. By contrast, in normal cells DsRed signals were detected in only 6 to $9 \%$ of the EGFP-positive cells. As expected, when the expression vector containing the TK promoter was used, no 

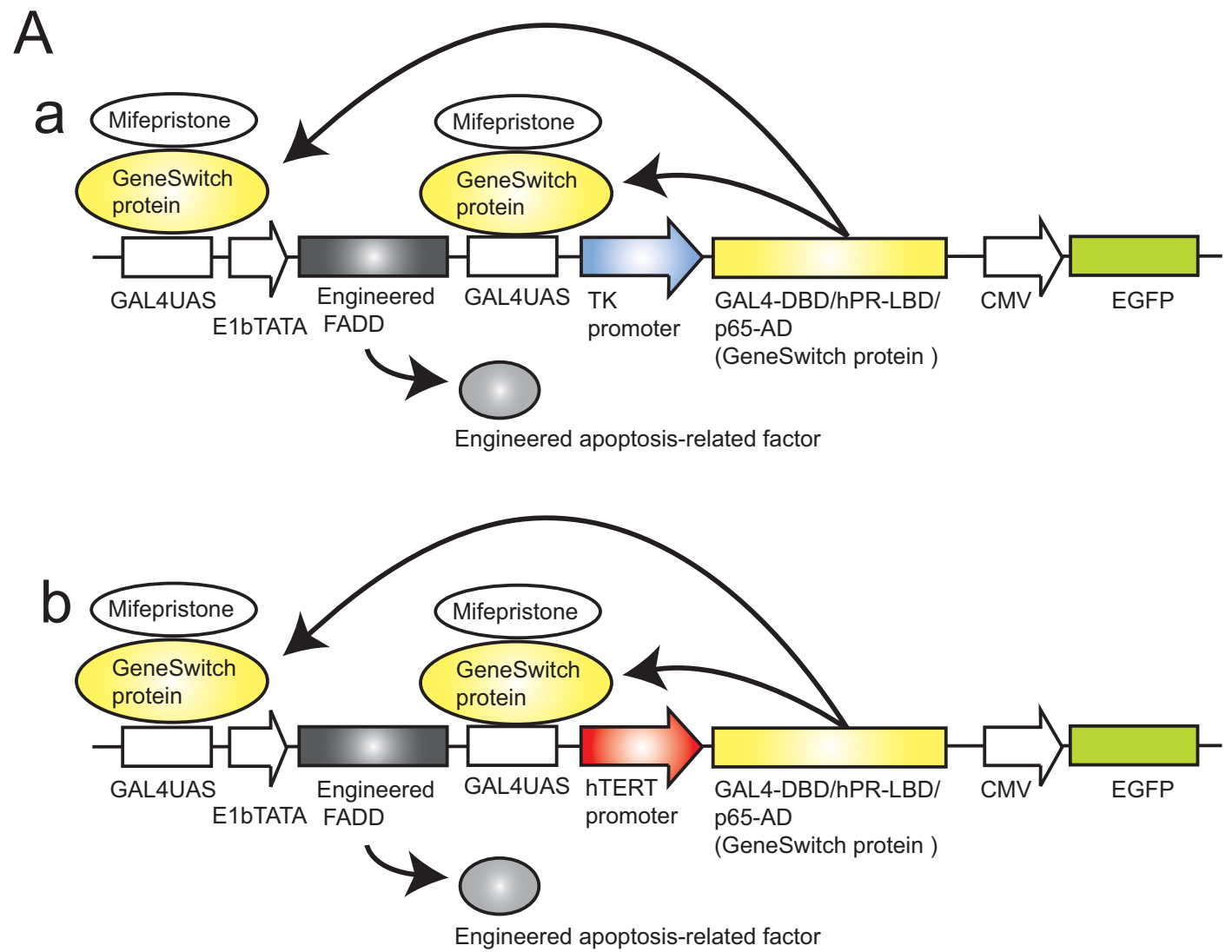

B

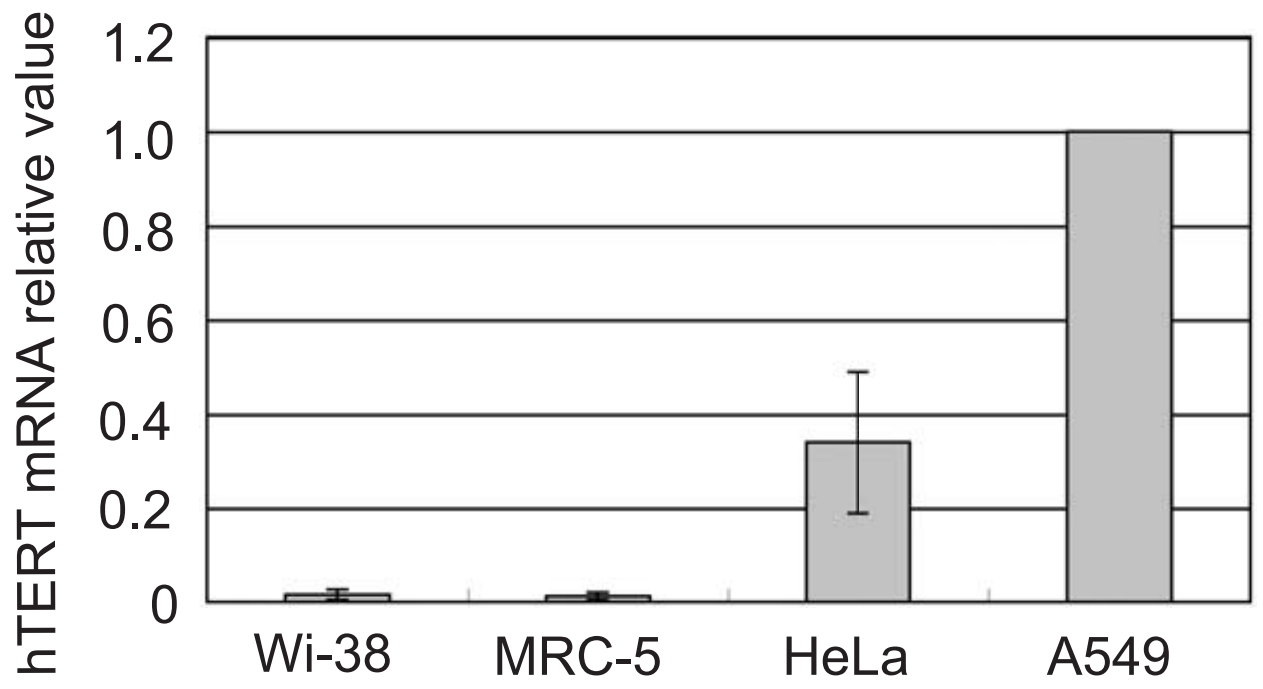

Fig. (3). (A) Schematic overview of a tumor-specific expression system based on an hTERT promoter and a mifepristone-dependent autoamplified expression system. Two promoters-a constitutive TK (Herpes thymidine kinase) promoter (a) and tumor-specific hTERT promoter (b) - were used for the mifepristone-dependent transcription factor-mediated expression of the engineered apoptosis-related factors. Upon mifepristone treatment, either an engineered factor or DsRed was expressed indirectly via GAL4 DBD/hPR-LBD/p65-AD. EGFP expression was directed by a constitutive promoter (CMV promoter). EGFP was used a marker for transfected cells. (B) Comparison of hTERT mRNA expressed in tumor cells and normal cells. The amounts of hTERT mRNA in tumor cells (HeLa and A549) and normal cells (Wi-38 and MRC-5) were measured by quantitative PCR. The amounts of hTERT mRNA are presented as relative values based on hTERT mRNA expression in A549 cells, which was set to one. Normal cells expressed low levels of hTERT mRNA, whereas tumor cells expressed relatively higher levels of hTERT mRNA. This is consistent with previous reports. 
significant difference was observed between tumor cells and normal cells (data not shown). TK promoter expression has been shown to occur at almost the same level in both normal cells and tumor cells.

On the basis of the results from the tumor-specific expression of the DsRed monitoring protein, we replaced the DsRed gene with 2DEDcasp8 $\triangle$ DEDa gene. The plasmid containing the 2DEDcasp8 $\triangle \mathrm{DEDa}$ gene was then transfected into tumor cells and normal cells, and the number of EGFPexpressing cells was then counted one day after transfection. Mifepristone was added to the cell culture medium to induce the expression of the engineered factor. Six hours after mifepristone induction, the number of surviving EGFPexpressing cells was re-counted. While no cell death was detected in both lines of normal cells (Wi-38 and MRC-5 cells), a significant level of cell death was detected in both lines of tumor cells (HeLa and A549 cells) (Fig. 4). By contrast, in normal and tumor cells transfected with a plasmid containing 2DEDcasp8 $\triangle \mathrm{DEDa}$ regulated by a constitutive TK promoter, almost the same level of cell death was detected in both types of cells. These results demonstrated that our vector system employing the hTERT promoter and GeneSwitch system distinguished tumor cells from normal cells, effectively inducing cell death in only tumor cells.

\section{DISCUSSION}

In this study, we generated novel engineered apoptosisinducing factors that can induce apoptosis even in cells lacking caspase-8. The most efficient engineered factor was 2DEDcaps8 $\triangle D E D a$, which is composed of tandem DEDs of FADD, DEDb of procaspase- 8 , and p18 and p10 of caspase8. Furthermore, we were able to express one of the engineered factors and induce apoptosis in a tumor cell-specific manner by using an expression vector containing a hTERT promoter in conjunction with the GeneSwitch system. With this system, tumor cells expressed high levels of the engineered factor, whereas normal cells expressed low levels of the engineered factor. Indeed, this expression system can distinguish tumor cells from normal cells, and then induce cell death effectively in only tumor cells.

Although 2DEDcaps8 and 2DEDcaps8 $\triangle$ DEDa are basically composed of tandem DEDs of FADD and procaspase8 , the apoptosis-inducing activity of 2DEDcaps 8 and 2 DEDcaps $8 \Delta$ DEDa was stronger than that of procaspase- 8 alone. This is consistent with our previous observations that 2DED strongly induces apoptosis [2]. Procaspase- 8 is inactive and requires activation by sequential signals, including Fas ligand-dependent induction of DISC (which is composed of FADD and procaspase-8). In the case of 2DEDcasp8 and 2DEDcaps $8 \triangle \mathrm{DEDa}$, the tandem DEDs of FADD mimic part of DISC formation. The DED of FADD binds to the DED of procaspase-8, and one of the tandem DEDs of 2DEDcasp8 binds to DEDb of the second 2DEDcasp8. Another tandem DED of 2DEDcasp8 binds the third 2DEDcasp8. These double binding sites can lead to the formation of 2DEDcasp8 multimers. It is should be noted that DED of FADD is also capable of self-dimerization activity. The engineered factor 2DEDcasp8 is already in an activated state and thus does not require death signals for activation.

Human telomerase reverse transcriptase (hTERT) is the catalytic subunit of telomerase, which is highly active in immortalized cells and $>85 \%$ of human cancers but is silent in most normal somatic cells [8, 9]. For tumor-specific transgene expression in cancer gene therapy, several groups have evaluated hTERT promoter activity with certain reporter genes driven by the hTERT core promoter [5]. Our expression system was made by incorporating the hTERT core promoter into the GeneSwitch expression system, the expression of which depends on the addition of mifepristone. In vitro, the GeneSwitch system provides ligand-dependent transgene expression in transfected cells, whereas in vivo it provides transgene expression controlled through orally administered mifepristone/RU-486. We used a modified version of the GeneSwitch system, one in which a transgene is not driven directly by the hTERT core promoter, for three reasons: (1) ligand dependency, (2) lowest basal expression

\section{Tk promoter}

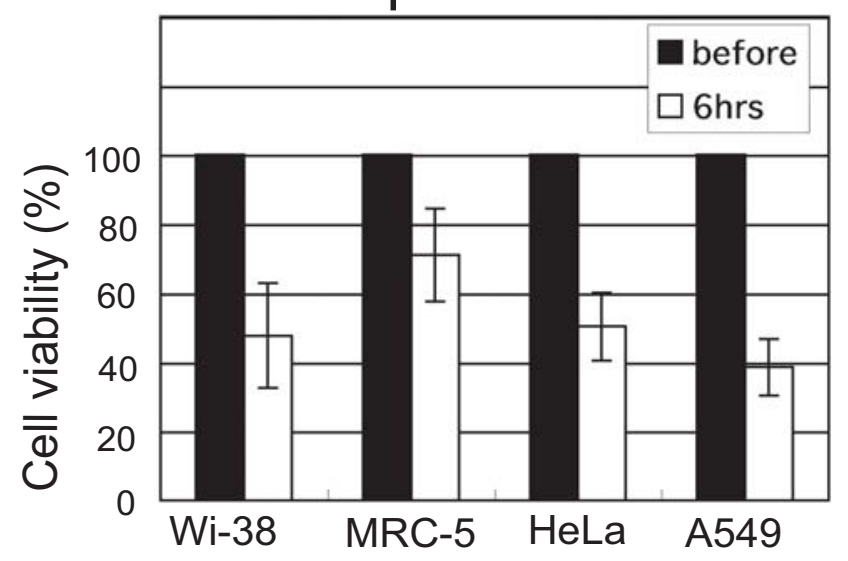

hTERT promoter

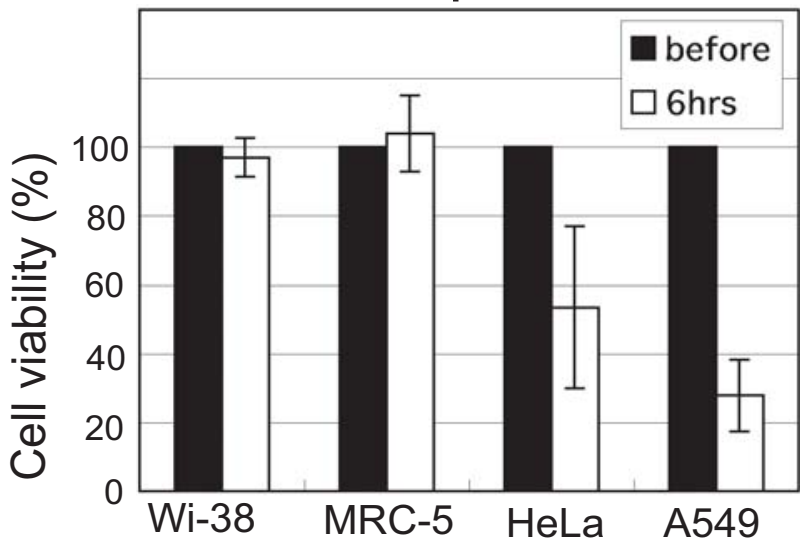

Fig. (4). Tumor-specific cell death in cells in which 2DEDcasp8 $\triangle D E D a$ expression is regulated by the hTERT promoter. Bar graphs showing the survival ratios of normal cells (Wi-38 and MRC-5) and tumor cells (HeLa and A549) transfected with 2DEDcasp8DDEDa 6 hours after mifepristone induction. (A) In cells in which 2DEDcasp8 $\triangle$ DEDa expression was regulated by a constitutive TK promoter, cell death was induced in both tumor cells and normal cells to almost the same levels. (B) In cells in which 2DEDcasp8DDEDa expression was regulated by the hTERT promoter, cell death was induced only in tumor cells. Normal cells were not affected. 
of the transgene without a ligand, and (3) auto-inducible amplified transgene expression. With our modified hTERTGeneSwitch expression system, we can distinguish tumor and normal cells, and then specifically induce apoptosis in tumor cells. The success we have experienced thus far with this system makes it a promising tool for future use in more complex systems, such as primary cultures derived from mixed tumor and normal tissue or in vivo models.

In the present study, we noticed potential problems with the hTERT-GeneSwitch expression system that needs to be resolved first before the system is applied to experimental paradigms having a more complex organization. Firstly, induced apoptosis efficiency is not optimal and should be improved toward $100 \%$ in order to avoid the re-expansion of surviving tumor cells. Comparison of results from our previous experiments on HeLa cells led us to speculate that low induced apoptosis efficiency is mainly due to relatively low levels of engineered factor expression. Circumventing this problem requires amplification of expression level in addition to our hTERT-GeneSwitch combinational expression system. Secondly, some tumor cells (e.g., Saos-2a cells) express low levels of the hTERT gene; thus, these tumor cells cannot express our engineered factors through the hTERTGeneSwitch expression system. Eliminating tumor cells that express low levels of hTERT would require the engineered factors to be generated through another promoter (e.g., AFP) [10]. Thus, in this regard, various promoters need to be tested. The double use or combinational use of such promoters will lead to the complete elimination of various tumors. Lastly, the method we used for transfecting our engineered factors for expression in cells is not optimal. A highly efficient method is required, such as one employing virus vectors (e.g., adenovirus vector) [5]. A more efficient delivery method will make our ligand-dependent tumor-specific expression system safer for use in vivo.

\section{ACKNOWLEDGEMENTS}

We are grateful to Emiko Suzuki and Sachiko Minorikawa for their excellent technical assistance. We also thank Dr. Osamu Ohara for his encouragement. This study was supported by grants from the Kazusa DNA Research Institute, and in part, by Grants-in-Aid for Scientific Research (KAKENHI) from the Japan Society for the Promotion of Science (JSPS).

\section{REFERENCES}

[1] Takashina T, Nakayama M. Modifications enhance the apoptosisinducing activity of FADD. Mol Cancer Ther 2007; 6: 1793-1803.

[2] Suzuki E, Takashina T, Nakayama M. Engineered FADD induces apoptosis via artificial death-inducing signaling complex (DISC). Int J Biomed Sci 2009; 5: 237-245.

[3] Brown JM, Attardi LD. The role of apoptosis in cancer development and treatment response. Nat Rev Cancer 2005; 5: 231-7.

[4] Stupack DG, Teitz T, Potter MD, et al. Potentiation of neuroblastoma metastasis by loss of caspase-8. Nature 2006; 439: 95-9.

[5] Murofushi Y, Nagano S, Kamizono J, et al. Cell cycle-specific changes in hTERT promoter activity in normal and cancerous cells in adenoviral gene therapy: a promising implication of telomerasedependent targeted cancer gene therapy. Int J Oncol 2006; 29: 6818.

[6] Kischkel FC, Lawrence DA, Tinel A, et al. Death receptor recruitment of endogenous caspase-10 and apoptosis initiation in the absence of caspase-8. J Biol Chem 2001; 276: 46639-46.

[7] Takita J, Yang HW, Bessho F, et al. Absent or reduced expression of the caspase 8 gene occurs frequently in neuroblastoma, but not commonly in Ewing sarcoma or rhabdomyosarcoma. Med Pediatr Oncol 2000; 35: 541-3.

[8] Kim NW, Piatyszek MA, Prowse KR, et al. Specific association of human telomerase activity with immortal cells and cancer. Science 1994; 266: 2011-15.

[9] Shay JW, Bacchetti S. A survey of telomerase activity in human cancer. Eur J Cancer 1997; 33: 787-91.

[10] Parks LC, Baer AN, Pollack M, Williams G.M. Alpha Fetoprotein: An index of progression or regression of hepatoma, and a target for immunotherapy. Ann Surg 1974; 180: 599-605. 